\title{
Repercusiones Multiculturales en el Desarrollo Lingüístico: Efectos en el Rendimiento
}

\author{
Francisco Mateos Claros $^{1}$ \\ Francisco Javier Olmedo Ruiz' \\ Macarena Esteban Ibáñez ${ }^{2}$ \\ Luis Amador Muñoz ${ }^{2}$ \\ ${ }^{1}$ Universidad de Granada \\ ${ }^{2}$ Universidad Pablo de Olavide de Sevilla
}

\begin{abstract}
Resumen
Este trabajo tiene como objetivo verificar las diferencias de rendimiento académico del alumnado de segundo ciclo de Educación Infantil que pertenecen a un contexto multicultural en el que coexisten una lengua oficial (castellano) y un dialecto cultural (darija). La muestra la componen un total de 501 alumnos cristianos y musulmanes del tercer curso a los que se les aplicó un cuestionario que evalúa el grado de adquisición de los aspectos competenciales de las áreas de comunicación y lenguaje, social y motriz. Para el análisis del conjunto de los datos se utilizaron pruebas estadísticas descriptivas y de contraste de variables. Los resultados demuestran como la lengua materna es un factor principal de diferenciación en todas las áreas del desempeño académico en este periodo, destacando las dimensiones comunicativas. Estas diferencias tienen una relación directa con la adhesión identitaria de los musulmanes a un dialecto cultural.

Palabras claves: Cultura; lenguaje; rendimiento académico; educación infantil
\end{abstract}

Repercussões Multiculturais no Desenvolvimento Linguístico: Efeitos no Desempenho Acadêmico

\begin{abstract}
Resumo
Este trabalho tem como objetivo verificar as diferenças no desempenho acadêmico dos alunos em segundo ciclo de Educação Infantil pertencentes a um contexto multicultural em que coexistem uma língua oficial (castelhano) e um dialeto cultural (Darija). A amostra foi composta por um total de 501 estudantes cristãos e muçulmanos no terceiro ano em que foram aplicados um questionário que avalia o grau de aquisição de questões jurisdicionais nas áreas de comunicação e linguagem, social e motor. Para a análise do conjunto de dados, foram utilizados testes descritivos e de contraste das variáveis. Os resultados mostram como a língua materna é um importante fator de diferenciação em todas as áreas de desempenho acadêmico durante este período, com destaque para a dimensão de comunicação. Essas diferenças têm uma relação direta com a adesão dos muçulmanos a um dialeto cultural.

Palabras clave: Cultura; linguagem; desempenho acadêmico; educação infantil
\end{abstract}

Multicultural Repercussions in Linguistic Development: Effectson Academic Performance

\begin{abstract}
The objective of this work is to verify the differences in the academic performance of students in the second cycle of Early Childhood Education who belong to a multicultural context in which an official language (Castilian) and a cultural dialect (darija) coexist. The sample is made up of a total of 501 Christian and Muslim students of the third year who were given a questionnaire that assesses the degree of acquisition of the competence aspects of the areas of communication and language, social and motor. For the analysis of the data set, descriptive and contrast tests of variables were used. The results demonstrate how the mother tongue is a main factor of differentiation in all areas of academic performance in this period, highlighting the communicative dimensions. These differences have a direct relationship with the adherence of Muslims to a cultural dialect.

Keywords: Culture; language; academic performance; early childhood education
\end{abstract}

\section{Introducción}

En el inicio de la educación reglada, en concreto la de Educación Infantil, tiene una implicación directa con un avance en el desarrollo de las habilidades cognitivas, comunicativas, afectivas y sociales que comprende una serie de cambios que se van produciendo en cada una de estas áreas lo que se conoce como desarrollo evolutivo. Un proceso que demanda la observación de múltiples factores que varían de forma sucesiva y simultánea, condicionándose entre sí y ajustándose al mundo externo (Vygotsky, 1996). Estos avances pueden ser definidos como conservación de Piaget (1988) o estructuras de comprensión (Wild, 2011) establecidos por "unas estructuras que tienen un desarrollo evolutivo, pero que el tiempo que necesita cada niño/a para crearlas depende de las circunstancias en las que vive" (p. 30). 
Por lo tanto, la evaluación debe hacerse de acuerdo con las características y los procesos evolutivos de los niños, teniendo en cuenta el uso de diferentes fuentes de información, partiendo de la universalidad de los aprendizajes a través de la igualdad de oportunidades (Mora, 2013). De ahí la importancia de hacer una valoración de las distintas áreas del desarrollo (cognitiva, afectiva, lenguaje, social y motricidad) y de su relación con la cultura (De la Peña, Parra y Fernández, 2018). Sin duda, la premisa principal será conseguir que el desarrollo del niño sea acorde a su edad y que le capacite para afrontar con preparación los distintos retos a través de una buena estructuración del aprendizaje.

Uno de los factores principales que interfieren en este escenario es el acercamiento de personas y el constante intercambio cultural. Por ello, diferentes modelos intentan explicar la adquisición, los efectos y el desarrollo de las competencias interculturales (Spitzberg y Changnon, 2009), partiendo de la base de que nos encontramos ante un hecho diverso e interconectado difícil de conocer en toda su extensión. Por lo tanto, la institución educativa se convierte en el principal escenario para fomentar la igualdad de estos nuevos contextos educativos (Caparrós y Casares, 2014). Según García y Moreno (2014) esta heterogeneidad existente en las aulas puede desencadenar discrepancias individuales que coexisten en todo el alumnado.

Esta situación que demanda la atención a diferentes culturas en este ámbito desde diferentes enfoques, pensamientos o ideologías, pudiendo oscilar desde una negación de las diferencias hasta su valoración como recurso educativo y social. Las aportaciones de Dickinson, Hofer, Barnes y Grifenhagen (2014) proponen que, en este entorno, se diseñen acciones educativas eficaces en el tratamiento de grupos diversos culturalmente, teniendo en cuenta sus características sociolingüísticas, así como a patrones de interacción y comunicación familiar. De hecho, el grupo étnico, el origen social, la lengua, etc. focalizan la cultura como variable educativa y la diversidad como norma, consideraciones que determinan la importancia del entorno directo en los logros educativos (Marjoribanks, 2005).

Razón por la cual, el ámbito familiar es el principal medio que condicionará la adquisición del lenguaje natural y su evolución (Yoshikawa, 2015), claramente influenciado por un modelo lingüístico que puede interferir en el avance escolar en el periodo infantil (Olmedo, 2017). Estas interacciones verbales son el punto de partida de los procesos de enseñanza-aprendizaje y el avance de funciones psicológicas superiores (Vygotsky,
1996), que en ocasiones puede estar determinado por un modelo cultural que puede influir en el avance de las distintas dimensiones del desarrollo evolutivo (De la Peña et al., 2018).

Por ello, la Educación Infantil se convierte en uno de los cambios más significativos de la infancia por la transición del contexto familiar al escolar (Chan, 2010), además de un periodo crítico para la incorporación a la Educación Primaria, la reducción del fracaso y el abandono escolar temprano (Alemany, Rojas, Gallardo y Sánchez, 2013). De ahí la importancia de entender los distintos elementos que intervienen en el rendimiento académico, acentuando la influencia del entorno asociado a ambientes personales, familiares y sociales determinados por las características culturales (Olmedo, 2017). Asimismo, la convivencia y el hábitat en distintos entornos pueden generar diferentes estilos cognitivos, determinados por el modo de comunicación utilizado en las relaciones, partiendo del lenguaje como medio principal que en ocasiones tiene que responder a distintos modelos conceptuales según la cultura (Rodríguez, 2017). Desde esta perspectiva, puede decirse que la lengua es un fenómeno social, cuyo desarrollo incide necesariamente el entorno socioambiental y los instrumentos que este ámbito puede ofrecer para avanzar el progreso lingüístico y cognitivo del sujeto (Halliday, 1983).

Partiendo de la importancia del lenguaje en la formación y la socialización de la persona, y teniendo en cuenta la implicación de la familia en su desarrollo, es capital considerar los problemas en su dominio en el inicio asociados a carencias lingüísticas culturales que puedan perjudicar el avance de la lengua oficial (Olmedo, 2017).Los patrones familiares y la conciencia étnica en la transmisión de modelos de aprendizaje establecen una de las líneas principales a tener en cuenta a la hora de definir las variables que afectan al rendimiento escolar en contextos multiculturales (Latorre, Mateos, Olmedo y Esteban, 2018).

Para Bartolomé (2017), esta diversidad que caracteriza los ámbitos escolares nos presenta múltiples procedencias, culturas y religiones. Indiscutiblemente, la religión se convierte en uno de los aspectos más significativos de la interculturalidad, principalmente en zonas en las que pueda darse la convivencia entre distintos credos. Según Berger (1999) este contexto demanda una modernidad que potencie la pluralidad frente a la secularización en la defensa de la diversidad, sin excluir la defensa de la libertad religiosa y de expresión; la igualdad en las diferencias implica garantizar la pluralidad, la convivencia y el diálogo. 
Si a todo esto, le añadimos la adhesión de algunas culturas a la triangulación cultura-religión-lenguaje, nos encontramos que en muchas ocasiones con un bajo dominio de la lengua oficial-escolar. De ahí la importancia de potenciar la lengua vehicular-oficial en este periodo en el cual su enseñanza se convierte en un elemento esencial para los avances educativos (González y Martín, 2017; Turner y Cross, 2016).

Llegados a este punto, nuestro objetivo se va a centrar en verificar que las diferencias en el progreso académico en el periodo de Educación Infantil están influenciadas por de un dialecto cultural no oficial (musulmanes) que interfiere de forma negativa en los alumnos de lengua materna darija, independientemente del género al que pertenecen. Se buscó contribuir a la comprensión de la influencia que estos resultados obtenidos representan la realidad a la que se refieren.

\section{Método}

Esta investigación está realizada con una metodología descriptiva, de carácter transversal y cuantitativo. Para ello se evaluaron competencias básicas asociadas a este periodo. En consecuencia, y con la intención de conocer la discrepancia de estos alumnos, las comparaciones se ajustaron a las variables género y lengua materna.

\section{Participantes}

Para la unidad muestral de los participantes de este estudio (alumnado de Educación Infantil de segundo ciclo) se tuvo en cuenta una representatividad de los centros educativos de este ámbito que pudiesen plasmar sus peculiaridades. El método elegido para la selección de los sujetos del estudio se ha basado en la aplicación de un muestreo aleatorio, con la intención de obtener las singularidades que representa este espacio multicultural, teniendo en cuenta las siguientes variables a la hora de hacer los estratos: público-concertado, centro-extrarradio, caracterización sociolingüística, etc. La muestra se seleccionó de una población de 1277 alumnos pertenecientes al tercer curso del segundo ciclo de infantil, de ellos se obtuvo una muestra productora de 501 alumnos, de los cuales, el $54,76 \%$ eran de género masculino $(n=322)$ y el $45,24 \%(n=299)$ de género femenino. En cuanto a la lengua materna, el 36,12\% $(n=259)$ eran castellanos parlantes y el 63,88 \% $(\mathrm{n}=362)$ darijo parlantes. De estos, el 58,08\% (n=291) tenían 5 años y el $41,91 \%(n=210) 6$ años.

\section{Instrumento}

La elaboración del instrumento se realizó a partir de la revisión de distintas fuentes bibliográficas, mencionadas con anterioridad, según características, elementos que lo definen, diferencias y evolución (Piaget, 1988; Vygotsky, 1996; Wild, 2011). Para ello se han comparado distintos instrumentos estandarizados como el WISC, Merrill Palmer R (Roid y Sampers, 2011), pruebas pedagógicas graduadas, etc., añadiendo cuestiones para puntualizar la información.

El cuestionario consta de 55 ítems que se responden a través de una escala de Likert de 4 categorías ( 1 =nunca, $2=$ a veces, $3=$ a menudo, $4=$ siempre $)$ en el que se recoge el grado de adquisición de aspectos competenciales básicos de esta etapa. Las distintas variables comprenden el área de comunicación y lenguaje (comprensión oral, expresión oral, expresión escrita, conciencia fonética, y lenguaje matemático) que cuenta con 30 ítems; el área de habilidades sociales (desarrollo emocional, la actitud ante los compañeros, la actitud ante el trabajo, ante el juego y hábitos) en la que se agrupan 19 ítems y finalmente el área motriz con 11 ítems. Para medir la fiabilidad del instrumento se utilizó el cálculo estadístico Alpha de Cronbach, una de las pruebas más utilizadas en investigaciones psicométricas (Maroco \& García, 2013), mostrando una consistencia interna total muy aceptable (.959) que se cumple igualmente en la definición de la escala comunicativa (.960), de las habilidades sociales (.791) y de la motricidad (.871). La validez de constructo se realizó a través de un análisis factorial exploratorio (KMO .959 y la prueba de Bartlett .000) con la intención de medir la estructura común subyacente en los datos valorados.

\section{Análisis de datos}

Para el análisis se utilizó el paquete estadístico SPSS versión 22, con el propósito de examinar estadísticos descriptivos y estadísticos de contraste. La intención principal estaba centrada en poder examinar en qué modo, estructura y grado eran dependientes entre sí las variables consideradas en nuestro estudio, teniendo en cuenta las funciones principales que deben cumplir los procedimientos de la estadística descriptiva (Fox, 1981). En primer lugar, el resultado de la prueba de Kolmogorov-Smirnov arrojó un nivel de significación (.123) que verifica la existencia de normalidad. Esta prueba nos permitió utilizar la prueba de comparación de medias ANOVA (paramétrica). Seguidamente, las frecuencias de porcentajes nos permitieron presentar la distribución de los alumnos según la lengua materna y 
el periodo de estudio. En cambio, para las medias de rendimiento de estos alumnos por periodo y lengua maternas se utilizó un gráfico de barras. Finalmente, los estadísticos de contraste utilizados nos admitieron conocer la existencia de diferencias significativas en el rendimiento académico de estos niños según el género y la lengua materna.

\section{Procedimiento}

Anterior a la recogida de datos se obtuvo el consentimiento informado por parte del MECD. El instrumento se repartió entre los docentes de tercer curso de Educación Infantil para ser cumplimentados, previamente fueron informados de su carácter anónimo, confidencial y voluntario, además de aclarar posibles dudas que pudieran surgir a la hora de contestar los ítems de cada alumno. Este proceso se desarrolló en el mes de mayo por ser el periodo más aproximado a la finalización del curso escolar. El tiempo estimado para responder el instrumento osciló entre 15 y 25 minutos por alumno. Antes de su finalización se verificó en la recolección que todos los ítems estuvieran contestados.

\section{Resultados}

Los estadísticos descriptivos utilizados en el tratamiento de los datos estuvieron centrados en conocer la distribución de las variables según su comparación, representados a través de una tabla de elaboración específica en las que aparecen representados los datos con significación.

En primer lugar, en la tabla 1 se representan los resultados de las puntuaciones de las distintas áreas de rendimiento en comunicación y lenguaje según la lengua materna. Para ello, los datos observados se basan en un nivel de significancia de $\mathrm{p}<.05$, en un valor de $\mathrm{F}$, superior al baremo mínimo $(3,84)$, donde $\mathrm{F}=(3,843)$. El valor de la media más destacado se identifica en el grupo de los castellanos parlantes para la categoría describir fotos $(3,78)$, siendo el de los niños $(3,15)$ de la variable expresión escrita. A su vez, observamos que existen diferencias significativas entre todas las categorías, distribuidas en su totalidad con un valor superior a $F>(3,84)$ con un valor de $p<.001$, destacando $F$ $=(60,036)$ de la categoría describir fotos "expresión escrita". Datos que avalan la heterogeneidad entre los

Tabla 1.

ANOVA comunicación y lenguaje según la lengua materna y el género

\begin{tabular}{|c|c|c|c|c|c|c|c|c|c|}
\hline \multirow{2}{*}{$\begin{array}{l}\text { Lengua } \\
\text { materna }\end{array}$} & \multicolumn{4}{|c|}{ Media } & \multirow{2}{*}{ Género } & \multicolumn{4}{|c|}{ Media } \\
\hline & $\mathrm{CP}$ & $\mathrm{DP}$ & $\mathrm{F}$ & Sig. & & $\mathrm{H}$ & $\mathrm{M}$ & $\mathrm{F}$ & Sig. \\
\hline $\begin{array}{l}\text { Vocabulario } \\
\text { preciso }\end{array}$ & 3,71 & 3,17 & 50,414 & $* * *$ & Escucha cuentos & 3,34 & 3,55 & 5,470 & $* *$ \\
\hline $\begin{array}{l}\text { Utiliza } \\
\text { imaginación }\end{array}$ & 3,56 & 3,04 & 38,343 & $* * *$ & Se distrae & 2,56 & 2,18 & 17,998 & $* * *$ \\
\hline $\begin{array}{l}\text { Termina } \\
\text { cuentos }\end{array}$ & 3,55 & 3,07 & 32,440 & $* * *$ & Seguir narración & 3,18 & 3,45 & 11,597 & $* *$ \\
\hline $\begin{array}{l}\text { Describe } \\
\text { fotos }\end{array}$ & 3,78 & 3,15 & 60,036 & $* * *$ & Prod. comprensibles & 3,38 & 3,56 & 6,135 & $*$ \\
\hline Seguir diálogo & 3,75 & 3,34 & 30,874 & $* * *$ & Escribe dictan & 3,13 & 3,33 & 5,063 & $*$ \\
\hline Escribe dictan & 3,49 & 3,04 & 22,182 & $* * *$ & Dividir palabra sonido & 3,22 & 3,47 & 8,174 & $* *$ \\
\hline $\begin{array}{l}\text { Diferencia } \\
\text { palabra }\end{array}$ & 3,46 & 3,07 & 20,605 & $* * *$ & Ordena numeración & 3,63 & 3,76 & 5,184 & $*$ \\
\hline $\begin{array}{l}\text { Dibujo } \\
\text { palabra }\end{array}$ & 3,55 & 3,04 & 32,144 & $* * *$ & Copia triángulo & 3,71 & 3,83 & 5,010 & $*$ \\
\hline $\begin{array}{l}\text { Vocales } \\
\text { iguales }\end{array}$ & 3,43 & 2,97 & 22,471 & $* * *$ & Concepto lejos cerca & 3,59 & 3,78 & 10,024 & $* *$ \\
\hline
\end{tabular}

Nota. CP: castellano parlante; DP: Darijo parlante castellano; H: hombre; M: mujer; *valores significativos $p<.05$; ** valores muy significativos $p<.01 ; * *$ valores altamente significativos $p<.001$. 
grupos de lengua materna castellana y lengua materna darija, en todas las dimensiones.

Seguidamente, detallamos los resultados relativos a las puntuaciones en función del género en base a la influencia de la variable tomada como dependiente, "lenguaje y comunicación”, y sus categorías. Observando los datos de la tabla apoyamos la idea de que existen diferencias estadísticamente significativas entre los niños y las niñas con un valor de $p<.001$ para la categoría escucha cuentos (comprensión oral), con un valor de $p<.01$ para la categoría escucha cuentos-seguir narración (comprensión oral), dividir palabra (conciencia fonética) y concepto lejos cerca (lenguaje matemático); aparte, el valor de $F$ es superior al baremo mínimo $(3,84)$ en todas las categorías observadas.

En la tabla 2 se expresan los resultados del rendimiento académico en habilidades sociales según la lengua materna y el género de estos alumnos. En el primer caso, observamos que, para cada subgrupo que engloba el factor "habilidad social", las diferencias en la media de rendimiento son desiguales según sea la lengua materna del alumno castellana o darija. Estas diferencias de medias son estadísticamente significativas a un nivel de $<.001$ para cada una de las variables, a excepción de la categoría tiene iniciativa, cuya probabilidad de errar a la hora de afirmar que existen diferencias significativas en el rendimiento es de $<.01$. Como puede verse, los resultados en esta área de los alumnos de lengua materna castellana tienen un rango medio de 0,92 (valor máximo: 3,83 - valor mínimo: 2,91), mientras que los resultados en alumnos de lengua materna darija son de 0,93 (valor máximo: 3,49 - mínimo: 2,54) y, por lo tanto, se comprueba la menor variabilidad de resultados en el alumnado de lengua materna castellana.
Asimismo, la variable género vuelve a confirmar que las alumnas obtienen datos superiores en todas las categorías, destacando la significación de todas las dimensiones a un nivel de $<.001$, excepto termina las tareas con un $\mathrm{p}<.01$, destacando tranquilo organizado $F=(35,762)$ y cuida el material $F=(39,746)$. Del mismo modo que en el anterior, los datos arrojan un rango de medio de 0,95 (valor máximo: 3,62 - valor mínimo: $2,67)$ para las niñas, mientras que los resultados en los niños son de 1,2 (valor máximo: 3,38 - mínimo: 2,18).

Para finalizar, en la tabla 3 se describen los resultados de los valores medios en rendimiento en cuanto a la motricidad, en función de la lengua materna y el género. Según los resultados de la variable lengua materna podemos concluir que las medias de rendimiento en estudiantes castellano parlantes y darija parlantes son estadísticamente diferentes a un nivel de significación de $<.01 \mathrm{y}<.001$. A la hora de detallar cada comparación en el rendimiento motriz, se observa que el rendimiento es mayor entre aquellos alumnos de lengua materna castellana a excepción de la variable "movimientos compensados" cuyo resultado es favorable en alumnos/as de lengua materna darija. De nuevo, en todos los casos se evidencia que el rendimiento en motricidad está afectado por la procedencia de la lengua materna del alumnado.

En cuanto a la variable género, las medias de rendimiento en las niñas son superiores que las de los niños, con un nivel de significación de de $<.05$ en la categoría sensaciones corporales, $<.05$ en el manejo del lápiz y de $\mathrm{p}<.001$ para la regularidad en el trazo la y precisión recorte, estas últimas relacionadas con un desarrollo óptimo en la utilización de músculos más pequeños "motricidad fina".

Tabla 2.

ANOVA babilidades sociales según la lengua materna y el género

\begin{tabular}{|c|c|c|c|c|c|c|c|c|c|}
\hline \multirow{2}{*}{ Lengua materna } & \multicolumn{4}{|c|}{ Media } & \multirow{2}{*}{ Género } & \multicolumn{4}{|c|}{ Media } \\
\hline & $\mathrm{CP}$ & $\mathrm{DP}$ & $\mathrm{F}$ & Sig. & & $\mathrm{H}$ & $\mathrm{M}$ & $\mathrm{F}$ & Sig. \\
\hline Identifica emociones & 3,83 & 3,49 & 30,579 & $* * *$ & Acepta normas & 3,30 & 3,62 & 18,593 & $* * *$ \\
\hline Comparte sentimientos & 3,80 & 3,49 & 34,654 & $* * *$ & Perfeccionista & 2,58 & 2,98 & 17,301 & $* * *$ \\
\hline Acepta normas & 3,70 & 3,30 & 27,677 & $* * *$ & Tranquilo organizado & 2,18 & 2,67 & 35,762 & $* * *$ \\
\hline Ritmo de trabajo & 3,62 & 3,29 & 16,333 & $* * *$ & Ordena & 3,08 & 3,52 & 31,297 & $* * *$ \\
\hline Cuida material & 3,51 & 3,21 & 12,482 & $* * *$ & Cuida material & 3,09 & 3,59 & 39,746 & $* * *$ \\
\hline Tiene iniciativa & 2,91 & 2,54 & 10,560 & $* *$ & Termina tareas & 3,38 & 3,62 & 7,697 & $* *$ \\
\hline
\end{tabular}

Nota. CP: castellano parlante; DP: Darijo parlante castellano; H: hombre; M: mujer; *valores significativos $p<.05$; ** valores muy significativos $p<.01 ; * * *$ valores altamente significativos $p<.001$. 
Una vez definidas las diferencias en los niveles según género y lengua materna, claramente con valores superiores en las niñas y la lengua materna castellana, queremos corroborar el peso de la lengua en este proceso, por ello, y según los resultados anteriores, hemos valorado si existe significación estadística en las diferencias de las medias de los cuatro grupos de la variable factor a través de una ANOVA tomando como variable dependiente de referencias la lengua materna castellana de niños y la lengua materna darija de niñas, aunque para la representación de los datos hemos utilizado los cuatro grupos (ver tabla 4).

Con respecto a la tabla anterior (tabla 4), y atendiendo a las todas las dimensiones analizadas, podemos observar como en este caso se invierte la tendencia de los resultados en función del género, arrojando medias superiores a favor de los niños, en este caso los castellano parlantes y valores inferiores en las niñas de lengua materna darija. En consecuencia, en estos dos grupos, se observa valores más altos de F de Fisher (heterogeneidad) en las dimensiones actitud ante el trabajo $(F=16,050)$, seguida de la expresión oral $(F=12,259)$ y la expresión escrita $(F=11,758)$, por encima del valor mínimo requerido $(F=3,84)$ y todas con valores altamente significativos $p<.001$ menos la categoría actitud ante el juego con un valor $p<.01$. De modo similar, las diferencias de medias más destacadas están los grupos de niños castellano parlantes y

Tabla 3.

ANOVA motricidad según la lengua materna y el género

\begin{tabular}{|c|c|c|c|c|c|c|c|c|c|}
\hline \multirow{2}{*}{ Lengua materna } & \multicolumn{4}{|c|}{ Media } & \multirow{2}{*}{ Género } & \multicolumn{4}{|c|}{ Media } \\
\hline & $\mathrm{CP}$ & $\mathrm{DP}$ & $\mathrm{F}$ & Sig. & & $\mathrm{H}$ & $\mathrm{M}$ & $\mathrm{F}$ & Sig. \\
\hline Baja escaleras & 3,80 & 3,62 & 8,689 & *** & Manejo lápiz & 3,66 & 3,85 & 14,354 & $* *$ \\
\hline Mov. compensado & 2,24 & 3,02 & 46,443 & $* *$ & Precisión recorte & 3,45 & 3,68 & 14,812 & $* * *$ \\
\hline Nombra posiciones & 3,84 & 3,64 & 11,849 & $* *$ & Regularidad trazo & 3,34 & 3,63 & 16,812 & $* *$ \\
\hline Sensación corporal & 3,82 & 3,62 & 10,745 & $* *$ & Sensación corporal & 3,64 & 3,77 & 4,560 & * \\
\hline
\end{tabular}

Nota. CP: castellano parlante; DP: Darijo parlante castellano; H: hombre; M: mujer; *valores significativos $p<.05$; **valores muy significativos $p<.01 ; * * *$ valores altamente significativos $p<.001$.

Tabla 4.

ANOVA Rendimiento género y lengua materna mixta

\begin{tabular}{lcccccc}
\hline \multicolumn{1}{c}{ Lengua materna } & \multicolumn{7}{c}{ Media } \\
\cline { 2 - 7 } \multicolumn{1}{c}{ Género } & OCP & ODP & ACP & ADP & F & Sig. \\
\hline Comprensión oral & 3,66 & 3,21 & 3,72 & 3,36 & 11,372 & $* * *$ \\
Expresión oral & 3,56 & 3,14 & 3,74 & 3,28 & 12,259 & $* * *$ \\
Expresión escrita & 3,49 & 3,04 & 3,64 & 3,12 & 11,758 & $* * *$ \\
Conciencia fonética & 3,30 & 2,94 & 3,54 & 2,98 & 8,602 & $* * *$ \\
Lenguaje matemático & 3,63 & 3,28 & 3,74 & 3,46 & 8,629 & $* * *$ \\
Estado emocional & 3,83 & 3,45 & 3,91 & 3,60 & 11,282 & $* * *$ \\
Actitud compañeros & 3,77 & 3,45 & 3,72 & 3,52 & 6,279 & $* * *$ \\
Actitud trabajo & 3,70 & 3,17 & 3,79 & 3,42 & 16,050 & $* * *$ \\
Actitud juego & 2,84 & 2,42 & 2,86 & 2,75 & 5,890 & $* *$ \\
Hábitos & 3,52 & 3,21 & 3,72 & 3,45 & 8,515 & $* * *$ \\
Motricidad & 3,84 & 3,44 & 3,92 & 3,61 & 11,376 & $* * *$ \\
\hline
\end{tabular}

Nota. OCP: niño castellano parlante; ODP: niño darijo parlante; ACP: niña castelano parlante; ADP: niña darijo parlante; *valores significativos $p<.05 ; * *$ valores muy significativos $p<.01 ; * * *$ valores altamente significativos $p<.001$. 
niñas darijo parlantes muestran mejores datos para los niños de lengua materna castellana en las dimensiones de expresión escrita (- .37) y conciencia fonética (- .32). Nuevamente, esta situación refuerza el peso que tiene la lengua independientemente del género de estos alumnos.

Para finalizar, queremos reafirmar la influencia de la lengua materna en el rendimiento académico de estos alumnos mostrando los resultados de distintos centros educativos y periodos académicos. Para ello, hemos decidido mostrar una tabla donde está representado el número de alumnos de lengua materna castellana y lengua materna darija por centro educativo y periodos (ver tabla 5), una tabla de chi-cuadrado (tabla 6) que verifique que existe homogeneidad en los periodos con respecto al estudio, y finalmente hemos representado las medias de rendimiento académico a través de un diagrama de barra agrupado en función del primer periodo y el segundo periodo de cada centro educativo (figura 1 ).
Al revisar los datos de los alumnos de cada centro educativo según el periodo y la lengua materna (ver tabla 5) se evidencia que los porcentajes de los castellanos parlantes disminuyen del primer periodo al segundo en los centros educativos C1 (- 4,17\%); C2 (- 28,65\%); el C4 $(-24,19 \%)$; C8 $(9,92 \%)$ y C9 $(19,42 \%)$. En cambio, nos encontramos un aumento de alumnos castellanos parlantes en el segundo periodo de los centros C3 $(50,7 \%)$; C5 $(50 \%)$; C6 $(7,41 \%)$ y C7 $(32,11 \%)$. Igualmente, podemos observar como los centros con mayor porcentaje de alumnos darijo parlantes son el C5; C6 y C7 (100\%) para el primer periodo y el C9 $(94,42 \%)$; C6 $(92,59 \%)$ y C8 $(72,41 \%)$ en el caso del segundo periodo.

Asimismo, los datos de la tabla 6 avalan que existe heterogeneidad (.000) y una relación de influencia del primer periodo general y el segundo sobre el rendimiento académicos de cada centro educativo.

Ahora, examinaremos brevemente en la figura 1 las diferencias de los rendimientos académicos generales de cada uno de los centros en función del periodo

Tablas 5 .

Porcentajes de alumnos castellano parlantes y darijo parlantes por centro educativo y periodo

\begin{tabular}{lcccc}
\hline & \multicolumn{2}{c}{ AP1 } & AP2 \\
\cline { 2 - 5 } & LMC & LMD & 45,83 & LMD \\
\hline Centro educativo 1 & 50 & 50 & 34,61 & 54,17 \\
Centro educativo 2 & 63,26 & 36,74 & 67,44 & 32,39 \\
Centro educativo 3 & 16,66 & 83,33 & 39,59 & 63,41 \\
Centro educativo 4 & 15,4 & 84,46 & 50 & 50 \\
Centro educativo 5 & --- & 100 & 7,41 & 92,59 \\
Centro educativo 6 & -- & 100 & 32,11 & 67,89 \\
Centro educativo 7 & -- & 62,5 & 27,58 & 72,41 \\
Centro educativo 8 & 37,5 & 76 & 4,58 & 94,42 \\
Centro educativo 9 & 24 & & & \\
\hline
\end{tabular}

Nota. Lengua materna castellana= LMC; Lengua materna darija $=$ LMD; Alumnos periodo $1=\mathrm{AP} 1$; alumnos periodo $2=\mathrm{AP} 2$

Tabla 6.

Homogeneidad periodos de rendimiento

\begin{tabular}{cccc}
\hline & Valor & gl & Sig. asintótica (bilateral) \\
\hline Chi-cuadrado de Pearson & $501,000^{a}$ & 17 &, 000 \\
Razón de verosimilitudes & 660,941 & 17 &, 000 \\
Asociación lineal por lineal & 163,133 & 1 &, 000 \\
N de casos válidos & 501 & & \\
\hline
\end{tabular}




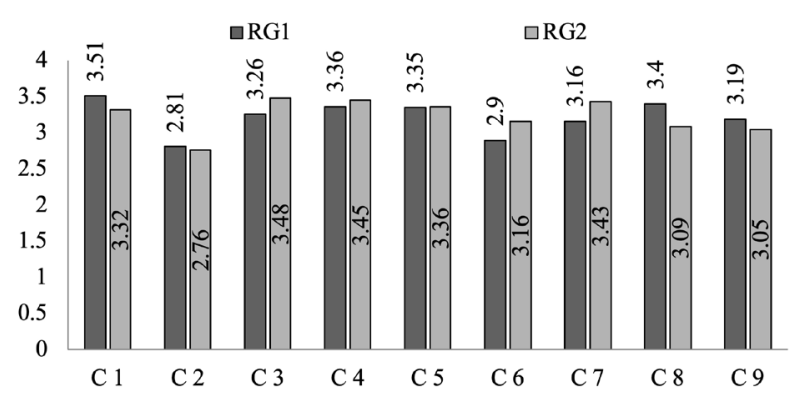

Nota. Rendimiento general primer periodo $=\mathrm{RG1}$; rendimiento general segundo periodo $=\mathrm{RG} 2 ; \mathrm{C}=$ centro educativo

Figura 1. Rendimiento académico general por periodos según lengua materna

En este sentido, los centros educativos C1 $(3,51)$; C4 $(3,36)$; C8(3,4) presentan mayores valores de media en rendimiento para el primer periodo y en el caso del segundo periodo C3 $(3,48)$; C4 $(3,45)$; C7 $(3,43)$. Seguidamente, destacan por su nivel más bajos por centros para el primer C7 $(3,16)$; C6 $(2,9)$; C2 $(2,81)$ y para el segundo periodo los centros educativos C8 (3,09); C9 $(3,05)$; C2 (2,76). Independientemente a los valores anteriores, según la tabla 5 podemos visualizar como en todos los centros existe una directriz común en los resultados de rendimiento académico general por periodo relacionada con el aumento de alumnos de lengua materna (darija). De manera que en el momento que estos aumentan, ya sea el primer periodo (C2, C4, C5 y C6) o el segundo (C1, C3, C7, C8 y C9), los niveles generales de rendimiento académico bajan, datos que muestran una relación directa entre la distribución de los alumnos en función de su lengua materna y las diferencias en los valores de rendimiento de los centros educativos.

\section{Discusión}

Una vez expuestos los datos, vamos a llevar a cabo los hechos que hemos deducido de nuestra investigación, atendiendo al planteamiento de la influencia del contexto multicultural en el desarrollo lingüístico y en el rendimiento académico en Educación Infantil. En relación con este estudio surgen los distintos argumentos que nos exige una explicación. Es por esto, que es preciso considerar el género y la lengua materna, esta última como una de las variables con mayor peso en la determinación de estas discrepancias.
En primer lugar, según las aportaciones de los datos, las niñas presentan un mejor rendimiento académico en dimensiones relacionadas con el desarrollo de aptitudes lingüísticas, habilidades sociales y motricidad. Razón por la cual existe una relación directa entre los mejores niveles de rendimiento académico y el género femenino, mostrando que en la mayoría de los casos las chicas logran resultados superiores, con la excepción de la capacidad de estar atento y concentrado, aunque en este caso el valor es inverso. Como consecuencia, las niñas poseen destrezas implícitas en su dominio de la lengua y del habla que favorece el desarrollo superior en habilidades lingüísticas y un desarrollo superior (Harris, 1977) De hecho, con anterioridad las investigaciones de Maccoby y Jacklin (1974) confirmaron que las niñas tienen mejores habilidades verbales que los niños. Del mismo modo, otros autores defienden que los niños tienen mejor rendimiento en matemáticas y las niñas en lenguaje (Studenska, 2011). Una habilidad comunicativa que interfiere en el avance de distintas capacidades relacionadas con habilidades sociales y motricidad. En particular, destacan en motricidad fina, habilidades que requieren movimientos detallados y en la coordinación óculo-manual de las niñas en agarre y la manipulación (Vidarte, Ezquerro y Giráldez, 2009).

Seguidamente, el análisis de rendimiento académico en función de la lengua materna presenta mejores resultados del alumnado castellano parlante que el darijo parlante en todas las áreas. En cuanto a la dimensión del lenguaje, la mayoría de las categorías muestran datos más positivos en aspectos relacionados con el proceso expresivo del lenguaje. Unas diferencias en su progreso que se asocian a una identidad marcada por la lingüística (Gineste, 2006). Así pues, la utilización de una lengua materna no estructurada (darija) ni asociada a la oficial merma la captación del mensaje y su posterior utilización. Querejeta (2017) destaca la importancia de la expresión escrita y la conciencia fonética en el proceso de lecto-escritura y como estas influyen en los logros académicos (Gómez, García, Vila, Elosúa y Rodríguez, 2014). Del mismo modo, las relaciones entre grafemas y fonemas que desarrollan los niños a través de la escritura de palabras les van a permitir fomentar habilidades que se pueden transferir a la lectura (Albuquerque y Alves, 2017).

Asimismo, esta diferencia en conciencia fonética a favor de los castellanos parlantes pone de manifiesto las consecuencias de un dialecto con un registro informal, de carácter oral no escrita (darija) y su sistema vocálico, interfiriendo en la utilización y empleo del canal 
auditivo en la diferenciación de fonemas en detrimento del futuro aprendizaje lector (Defior, 2014). En cambio, cuando diferenciamos la lengua, la universalidad del código matemático (Russell, 1919) hace que esta sea más semejante en su comparación por ser abstracciones que culturalmente están compartidas.

El mejor control externo de los alumnos de lengua materna castellana ante distintas situaciones en el ámbito escolar, al igual que una actitud positiva determinada hacia el sentido de cumplir con los demás, ante las normas y las obligaciones, revelan mejoras habilidades sociales que permite la evolución de conceptos relacionados con la mutualidad y reciprocidad (Olmedo, Mateos, Esteban y Trujillo, 2019). Una situación que se traslada al ámbito emocional, reflejando en los darijo parlantes unas capacidades inferiores en esta categoría, aspecto que podemos relacionar con la individualidad propia de su cultura. Para la definición del área motriz, nuevamente quedan corroboradas mejores habilidades de los alumnos de lengua materna castellana que potencian la coordinación de movimientos para un buen desarrollo y control motor, además de la organización y estructuración de su imagen corporal. De ahí que para Bryant y Cratty (1979) la relación entre las habilidades lingüísticas y motrices la convierten en una parte elemental del proceso educativo.

La importancia de la comunicación y el lenguaje toma consistencia cuando comparamos los resultados generales de los niños de lengua materna castellana con los de las niñas de lengua materna darija, donde podemos concretar que la tendencia que define la mayoritaria de los resultados en rendimiento académico según el género, que hasta el momento revelaron mejores resultados en las niñas que en los niños, cuando determinamos como base principal del rendimiento la lengua materna estos efectos se transforman. De esta manera, nuestro estudio revela que la lengua materna tiene mayor peso que el género (Olmedo, 2017) desmontando líneas de investigaciones que otorgan una relación directa entre el rendimiento escolar y el género (Pierart y Pavés, 2011).

De ahí que hayamos asociado el género con aspectos individuales y el lenguaje con factores externos (Vygotsky, 1996) partiendo de las influencias socioculturales del contexto de estudio, que en este caso tienen la lengua materna (darija) como elemento principal de la construcción del modelo comunicativo (Dickinson, Hofer, Barnes y Grifenhagen, 2014). En consecuencia, la influencia de este dialecto interfiere en lengua oficial, siendo esta, la base del crecimiento cognitivo y eje principal del proceso educativo (Luria y Yudovich, 1978).

Una lengua materna que marca discrepancia entre estos dos grupos y explica las diferencias de rendimiento académico general de los centros según los alumnos castellanos parlantes y darijo parlantes del segundo ciclo de Educación Infantil. Razón por la cual existe una relación directa entre el descenso del nivel de rendimiento académico y el aumento de alumnos con lengua materna darija en los centros educativos.

En definitiva, y en consonancia con todo lo anterior, la lengua materna darija se convierte en el principal factor que las distingue las diferencias en los niveles de rendimiento. Siendo esta también, la causa principal de las discrepancias en los rendimientos académicos generales de los centros educativos. Un entorno que influye de forma directa en las aptitudes necesarias para la consecución de los objetivos del periodo escolar de Infantil, cabe destacar, que nos encontramos ante periodo elemental que debe de garantizar un tránsito adecuado a la Educación Primaria que facilite su enlace (Castro, Ezquerra y Argos, 2018; Tamayo, 2014).

\section{Consideraciones finales}

En el presente estudio, independientemente a que los resultados verificaron nuestro objetivo, nos hemos encontrado con limitaciones en nuestra investigación. Destacamos que el estudio longitudinal (periodo o curso) de estos alumnos limita conocer los efectos en el rendimiento futuro o la evolución en cursos posteriores de esta muestra. Del mismo modo, el carácter multidimensional del rendimiento académico (García y Cantón, 2016) nos mostró divergencias para la demarcación, teniendo en cuenta que las variables interfieren y transfieren de unos contextos a otros. Una situación preestablecida que nos hizo trabajar con grupos naturales sin interferir en la distribución de los sujetos de cada grupo.

Igualmente, la edad de los alumnos de la muestra limitó el tipo de instrumento, independientemente a que fueron cumplimentados por los docentes. El cuestionario se caracteriza por la sencillez y rapidez para su formulación, pero el periodo para su aplicación resultó un inconveniente por carencias de tiempo de los docentes debido a que coincidió la finalización de etapa con la evaluación. Una vez observados los resultados queda claro la incidencia y la importancia del lenguaje en este ámbito multicultural. Por ello es preciso establecer futuros estudios que enriquezcan la investigación a través 
de la elaboración de un nuevo instrumento que nos permita constatar los resultados de un estudio longitudinal de la muestra del estudio, al menos en los tres primeros cursos de la siguiente etapa (primaria).

\section{Referencias}

Albuquerque, A. y Alves, M. (2017). Habilidades Iniciais de Alfabetização em Português: Pesquisa Transcultural em Portugal e no Brasil, Psico-USF, 23(3), 437-448. doi.org/10.1590/1413-82712017220305.

Alemany, I., Rojas, G., Gallardo, M.A. y Sánchez, S. (2013). El abandono escolar temprano en un contexto multicultural. Análisis de sus causas por los agentes profesionales y sociales implicados. Journal for Educators, Teachers and Trainers, 4(2), 191 - 203. Recuperado de: http://jett.labosfor.com/index. $\mathrm{php} /$ jett/article/view/75/75

Bartolomé, M. (2017). Diversidad educativa ¿Un potencial desconocido? Revista de Investigación Educativa, 35(1), 15-33. doi: 10.6018/rie.35.1.275031

Berger, P. (1999). The desecularization of the World. Resurgent Religion and World Politics. Washington: Ethics and Public Policy Center.

Bryant, J. y Cratty, B. (1979). Desarrollo perceptual y motor en los niños. Buenos Aires: Paidós.

Caparrós, N. y Casares, E. (2014). La escuela como elemento integrador: estudio de la eso en navarra. Revista Castellano-Manchega de Ciencias Sociales, 18, 137-146. doi: 10.20932/barataria.v0i18.48

Castro, A., Ezquerra, P. y Argos, J. (2018). Deepening the transition between childhood education and primary education: the perspective of families and teachers. Teoría de la Educación, 30(1), 217-240. doi. org/10.14201/teoredu301217240

Chan, W. (2010), “The Transition from Kindergarten to Primary School, as Experienced by Teachers, Parents and Children in Hong Kong". Early Child Development and Care, 180(7), 973-993. doi. org/10.1080/03004430802586130

Defior, S. (2014). Procesos implicados en el reconocimiento de las palabras escritas. Aula: revista de enseñanza e investigación educativa, 20, 25-44. Recuperado de: http://campus.usal.es/ revistas_trabajo/ index.php/0214-3402/article/view/12560/12878
De la Peña, C., Parra, N. y Fernández, J.M. (2018). Análisis de la alfabetización inicial en función del tipo de familia. OCNOS, 17(1), 7-20. doi. 10.18239/ ocnos_2018.17.1.1336

García, J. A. y Moreno, I. (2014). Escuela, Diversidad Cultural e Inclusión. Madrid: Catarata.

Gómez, I. M., García, J. A., Vila, J. O., Elosúa, M. R. \& Rodríguez, R. (2014). The Dual Processes Hypothesis in Mathematics Performance: Beliefs, Cognitive Reflection, Working Memory and Reasoning. Learning and Individual Differences, 29, 67-73. doi.org/10.1016/j.lindif.2013.10.001

Dickinson, D., Hofer, K., Barnes, E., \& Grifenhagen, J. (2014). Examining teachers' language in Head Start classrooms from a Systemic Linguistics Approach. Early Childhood Research Quarterly, 29(3), 231-244. doi:10.1016/j.ecresq.2014.02.006

García, S. y Cantón, I. (2016). Factores que influyen en el rendimiento académico. El camino hacia el éxito escolar. En J. L. Castejón (Coord.), Psicología y Educación. Presenta y futuro (pp. 2190-2198). Oviedo: España.

González, M.J., \& Martín, I. (2017). Effects on Reading of an Early Intervention Program for Spanish Children at Risk of Learning Difficulties. Remedial and Special Education. 38(2), 67-75. doi: 10.1177/0741932516657652

Fox, D. (1981). El proceso de investigación en Educación. Pamplona: EUNSA.

Halliday, M. (1983). Exploraciones de las Funciones del lenguaje. Barcelona: Paidós.

Harris, L. J. (1977). Sex differences in the growth and use of language. In E. Donelson \& J. Fullarhorn (Eds), Women: a psychological perspective (pp. 79-94). New York: Wiley.

Latorre, M.J., Mateos, F., Olmedo; F.J y Esteban, M. (2018). Modelos culturales en un contexto multicultural. International Journal of Educational Research and Innovation, 9, 31-45. Recuperado de: https://www.upo.es/revistas/ index.php/IJERI/article/view/2669/2223

Luria, A. R. y Yudovich, F. (1978). El papel del lenguaje en la formación de los procesos mentales. Infancia y aprendizaje, 3, 5-18. doi.org/10.1080/02103702.1 978.10821701. 
Maccoby, S. \& Jacklins, C. (1974). The psychology of sex differences. California: Stanford University Press.

Marjoribanks, K. (2005). Family and Children's Outcomes. Educational Psychology, 25 (6), 647-657.doi. org/10.1080/01443410500344704

Maroco, J. \& García, T. (2013). Qual a fiabilidade do alfa de Cronbach? Questões antigas e soluções modernas? Laboratório de Psicologia, 4 (1), 65-90. doi. org/10.14417/lp.763

Mora, F. (2013). Neuroeducación. Madrid: Alianza Editorial.

Olmedo, F.J. (2017). Cultura y Rendimiento del Alumnado de Segundo Ciclo de Educación Infantil en un Contexto Multicultural (Tesis Doctoral). Universidad de Granada, Granada.

Olmedo, F. J., Mateos, F., Esteban, M. y Trujillo, J. L. (2019). Indicadores culturales en el desarrollo de habilidades sociales del alumnado de infantil en contextos educativos diversos. En 7th International Congress of Educational Sciences and Development. Granada. España.

Piaget, J. (1988). Psicología evolutiva de Jean Piaget. México: Paidós.

Pierart, C. y Pavés, F. (2011). Estilos de aprendizaje, género y rendimiento académico. Revista Estilos de Aprendizaje, 8(8). Recuperado de: www.uned.es/ revistaestilosdeaprendizaje/numero_8/articulos/ 1sr_8articulo5.pdf

Querejeta, M. (2017). Conciencia fonémica y memoria fonológica en niños en proceso de alfabetización. Revista de Psicología, 16, 13-29. doi: 10.24215/2422572Xe003

Rodríguez, R. M. (2017). Cultura escolar vs cultura familiar: vincular escuela, familia y comunidad desde la pedagogía culturalmente responsiva. Interações, 43, 7-27. doi: 10.25755/int.12029

Russell, B. (1919). Introduction to Mathematical philosophy. London: George Allen and Unwin, Ltd.

Spitzberg, B. H. \& Changnon, G. (2009). Conceptualizing intercultural competence. In D.K. Deardorff (Ed.), The SAGE Handbookof Intercultural Competence (pp. 2-52). Thousand Oaks, CA: Sage.

Studenska, A. (2011). Educational level, gender and foreign language learning self regulation difficulty. Procedia-Social and Behavioral Sciences, 29, 1349-1358. doi.org/10.1016/j.sbspro.2011.11.373

Tamayo, S. (2014). La transición entre etapas educativas: de la Educación Infantil a Educación Primaria. Participación educativa, 5 (3), 131-137. Recuperado de: file:///C:/Users/tito_/AppData/Local/Packages/Microsoft.MicrosoftEdge_8wekyb3d8bbwe/ TempState/Downloads/16784\%20(2).pdf

Turner, M. \& Cross, R. (2016). Making space for multilingualism in Australian schooling. Language and Education, 30 (4), 289-297. doi.org/10.1080/0950 0782.2015 .1114627

Vygotsky, L.S. (1996). A farmação social da mente: o desenvolviendo dos procesos superiores. São Paulo: Martins Fontes.

Wild, R. (2011). Etapas del desarrollo. Barcelona: Herder.

Yoshikawa, H., Leyva, D., Snow, C. E., Treviño, E., Barata, M., Weiland, C., \& Arbour, M. C. (2015). Experimental impacts of a teacher profesional development program in Chile on preschool classroom quality and child outcomes. Developmental psychology, 51(3), 309-322. doi:10.1037/a0038785

Recebido em: 28/05/2018 Reformulado em: 25/02/2019 
Sobre os autores:

Francisco Mateos Claros (Universidad de Granada, España) Titular de Psicología Evolutiva y de la Educación de la Universidad de Granada. Investiga en: dificultades de aprendizaje, valores y actitudes de los jóvenes. Entre sus publicaciones: Las dificultades de aprendizaje en el contexto intercultural de Ceuta, Juventud Europea: Valores y Actitudes ante las instituciones democráticas.

ORCID: https://orcid.org/0000-0002-6596-9803

E-mail: fmateos@ugr.es

Francisco Javier Olmedo Ruiz (Universidad de Granada, España). Diplomado en Educación Social, licenciado en Psicopedagogía y Doctor en Modelos de Enseñanza-Aprendizaje y Desarrollo de las Instituciones Educativas por la Universidad de Granada. Ha participado en varios artículos y capítulos de libros y pertenece al Grupo de Investigación en Acción Educativa (HUM 929).

ORCID: https://orcid.org/0000-0002-0180-9288

E-mail: tito_javi75@hotmail.com

Macarena Esteban Ibáñez (Universidad Pablo de Olavide de Sevilla, España). Profesora del área de teoría de la Educación de Ciencias Sociales. Coordinadora del máster Interuniversitario en Educación Ambiental y autora de varios libros y artículos científicos sobre educación ambiental.

ORCID: https://orcid.org/0000-0002-2246-4784

E-mail: mestibañez@upo.es

Luis Amador Muñoz (Universidad Pablo de Olavide de Sevilla, España). Profesor titular de la Facultad de Ciencias sociales de la Universidad Pablo de Olavide. Dirige e imparte docencia en diversos doctorados y máster Oficiales. Ha participado y es autor de múltiples artículos, capítulos de libros y libros y miembro de la Comisión Andaluza de Educación Social

ORCID: https://orcid.org/0000-0003-2203-3236

E-mail:1vamador@upo.es

Contato com os autores:

Francisco Javier Olmedo Ruiz

C/ Real 86, Edificio Roma, Bloque $26^{a}$ A Ceuta

España

51001 